\title{
Rock microstructure in the deep extension of the Nagamachi-Rifu fault revealed by analysis of collocated seismic and magnetotelluric data: Implication of strong deformation process
}

\author{
Marina Pervukhina, Yasuto Kuwahara, and Hisao Ito \\ Geological Survey of Japan, National Institute of Advanced Industrial Science and Technology (AIST), Central 7, 1-1-1 Higashi, \\ Tsukuba, Ibaraki 305-8567, Japan
}

(Received July 13, 2004; Revised November 22, 2004; Accepted December 24, 2004)

\begin{abstract}
Quantitative analysis of collocated seismic velocity tomography and electromagnetic experiments is developed to elucidate the structure of the deep extension of the Nagamachi-Rifu fault, northeastern Japan. $P$ and $S$ wave seismic velocities obtained from a dense seismic network are examined and a ratio of spatial variation in $P$ and $S$ wave velocities $d \ln V_{s} / d \ln V_{p}$ is chosen as a proxy for the influence of pore geometry. The analysis shows that the deep extension of the Nagamachi-Rifu fault reveals the $d \ln V_{s} / d \ln V_{p}$ values exceeded 1.1. Such large values of $d \ln V_{s} / d \ln V_{p}$ cannot correspond to equilibrium pore geometry, at which the interfacial energy is at a minimum, and indicate regions with non-equilibrium state where non-isotropic stress prevents the equilibrium pore geometry to be achieved. To specify a fine distribution of porosity and connectivity of micropore in the region, we carry out the joint analysis of the seismic velocities with the electrical resistivity data obtained by the magnetotelluric survey crossing the Nagamachi-Rifu fault. It is shown that the region at $10-17 \mathrm{~km}$ depths at about $20-40 \mathrm{~km}$ to the northwest from the hypocenter of the M5.0 earthquake occurred in 1998 exposes the highest connectivity among the adjacent areas, suggesting a strong deformation process.

Key words: Active fault, seicmic velocity tomography, magnetotelluric experiment, fractal model, electrical and elastic properties of porous rock, Nagamachi-Rifu fault.
\end{abstract}

\section{Introduction}

Investigations of a deep structure of active faults have been recognized to be important, based on the hypothesis that an aseismic slip or localized shear zone in deep extensions of active faults beneath seismogenic zones controls the generation of large inland earthquakes (Iio, 1996; Iio and Kobayashi, 2002). The Nagamachi-Rifu fault is an active reverse fault running through the center of Sendai City, Northeastern Japan, in NE-SW direction. An earthquake of magnitude 5.0 occurred at the depth of $12 \mathrm{~km}$ about $10 \mathrm{~km}$ to the northwest of the Nagamachi-Rifu fault on 15 September 1998. Umino et al. (2002) suggested that this event was caused with a slip at a deep portion of the Nagamachi-Rifu fault.

Comprehensive studies of seismic, electromagnetic and GPS surveys have been performed to elucidate nature of the deep extension of the Nagamachi-Rifu fault. Nakamura et al. (2002) estimated deep geometry of the Nagamachi-Rifu fault from seismic array observations. Imanishi et al. (2002) tried to deduce seismic scatterer distribution in the region from small aperture seismic array observations. Nishimura et al. (2004) suggested aseismic sliding on a horizontal detachment connecting to the Nagamachi-Rifu fault in order to explain the data from continuous GPS stations across the

Copy right(c) The Society of Geomagnetism and Earth, Planetary and Space Sciences (SGEPSS); The Seismological Society of Japan; The Volcanological Society of Japan; The Geodetic Society of Japan; The Japanese Society for Planetary Sciences; TERRAPUB
Nagamachi-Rifu fault. Nakajima (2002) and Nakajima et al. (2004) estimated the three-dimensional seismic velocity structure in the region of the Nagamachi-Rifu fault by using a dense seismic network data. Ogawa et al. (2004) obtained electromagnetic image in the fault region. All the listed studies above separately analyzed the data obtained by different experiments, although all the types of experimental data might reflect the same deep structure. Thus joint analysis might be important to obtain reliable information about the deep structure.

Joint analysis of $P$ and $S$ wave velocities $\left(V_{p}\right.$ and $\left.V_{s}\right)$ and electrical resistivity of rock on the basis of an appropriate model should be useful for getting reliable conclusion about the microstructure and stress state of the region. Recent researches have clarified the plausible microstructure of porous rock in the middle and lower crust. Watson and Brenan (1987) showed that if a region is at an equilibrium state, i.e. at any point of the region the chemical equilibrium is achieved and the stress is isotropic, equilibrium pore geometry, at which interfacial energy is at a minimum, is reached at the temperature above $200-300^{\circ} \mathrm{C}$. According to Tanaka and Ishikawa (2002) the thermal gradient around the region of the deep extension of the Nagamachi-Rifu fault is more than $20^{\circ} \mathrm{C} / \mathrm{km}$, thus at the depth of $10 \mathrm{~km}$ the temperature is exceeded $200^{\circ} \mathrm{C}$. If equilibrium geometry has been achieved, fluids exist in channels along grain edges or collects at grain corners forming isolated pores. The geometry of liquid-filled pores is determined by the dihedral angle $\theta$. If $\theta<60^{\circ}$, the grain edge channel remain open, while 


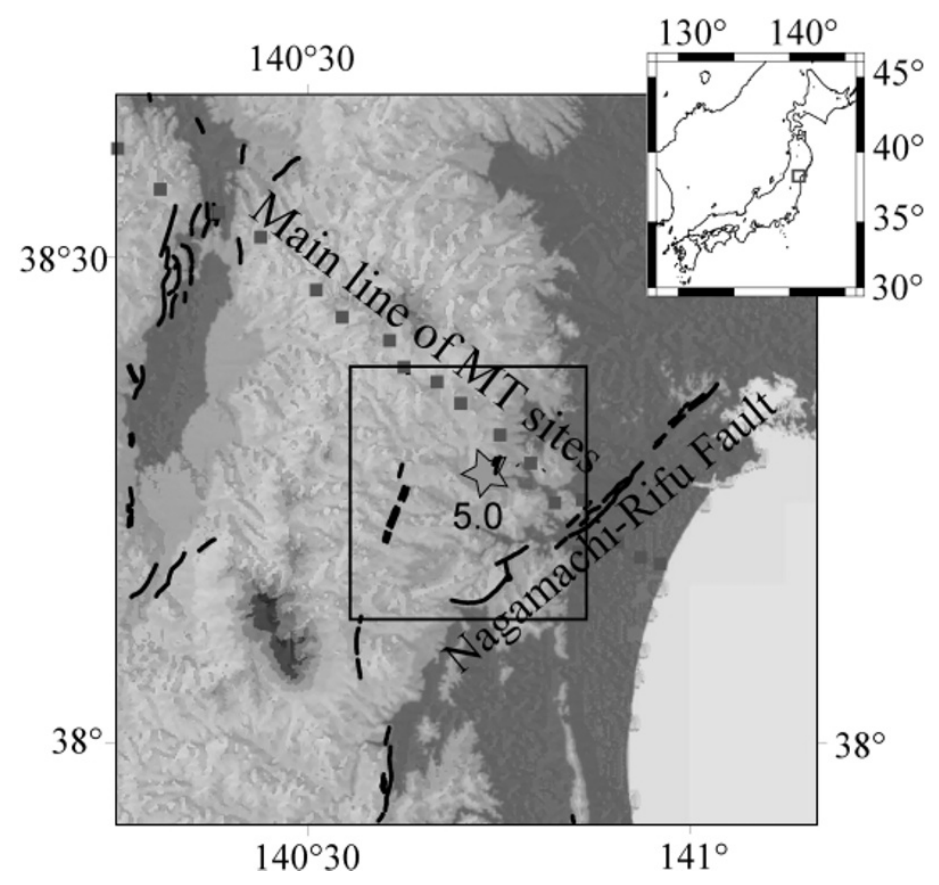

Fig. 1. Location of MT survey line (Ogawa et al., 2004) analyzed in the present study and the surface trace of the Nagamachi-Rifu fault. The epicenter of an earthquake with M 5.0, which occurred on this fault on 15 September 1998 at the depth of about $12 \mathrm{~km}$, is indicated by a star. Rectangle shows the area where the $P$ and $S$ wave velocity structures were estimated for grid nets spaced at intervals 5-7 km by Nakajima (2002).

if $\theta>60^{\circ}$, the channel will close off and fluid will form isolated pores. Hereafter, we will be concentrated on the results not for melt but for aqueous fluid, because the aqueous fluid has been suggested to present at the deep extension of the Nagamachi-Rifu fault (Nakajima, 2002; Nakajima et al., 2004). Having investigated different rocks and fluids of different compositions, Watson and Brenan (1987) showed that the dihedral angle ranges from $40^{\circ}$ to $100^{\circ}$ for rock-aqueous system. Hence, the lithospheric aqueous fluids can either exist as isolated pores or form a continuous interconnected system of channels. But in a region at equilibrium state the aqueous fluid cannot exist as films along grain boundaries, since existence of such films implies a dihedral angle of $\theta=0$. On the other hand, the fractures that develop as a result of tectonic processes or igneous intrusion do not expose the equilibrium pore geometry. These fractures provide alternative avenues of fluid transport that can form continuous films.

Takei $(1998,2002)$ developed a theoretical model to describe the equilibrium geometry as a function of pore aspect ratio. Takei (2002) showed that for rock-aqueous fluid system at 0-30 km depths, the equilibrium pore geometry corresponds to aspect ratio of $0.15-0.5$. She analyzed the influence of the microstructure on $d \ln V_{s} / d \ln V_{p}$, the ratio of the fractional changes in $P$ and $S$ wave velocities, and showed how to distinguish a region with the equilibrium geometry from the region which is under slip or shear stress. In a region with the equilibrium geometry that characterized with liquid-filled pores with aspect ratios $\alpha$ of $0.15-0.5$, the ratio $d \ln V_{s} / d \ln V_{p}$ is expected to be 0.8-1.1 (Takei, 2002). In a region with non-equilibrium pore geometry that is characterized by crack system with aspect ratios less than 0.15 , the water-rock system shows the larger values of the ratio $d \ln V_{s} / d \ln V_{p}$ such as 1.1-2.2 (Takei, 2002). Combining the results of Takei (2002) and Watson and Brenan (1987), we can conclude that the ratio $d \ln V_{s} / d \ln V_{p}$ might be an indicator of a degree of deformation.

To perform a joint analysis of both electrical and elastic properties, the fractal model of porous rock (Pervukhina et al., 2003, 2004) has been developed. The main advantage of this model is possibility to describe both elastic and electrical properties of rock with a single model for a wide range of microstructures including 3D grain and pore anisotropy and different interconnection extent, namely, isolated pores, partially interconnected ones, tubes and films. Thus, the analysis of electrical properties together with elastic properties provide us with more information of pore microstructure, especially pore connectivity, than that with the analysis only of the elastic properties.

In the present study, we first describe the experimental data of the collocated results of seismic velocity tomography by Nakajima (2002) and Nakajima et al. (2004) and electrical resistivity from the magnetotelluric survey crossing the Nagamachi-Rifu fault area by Ogawa et al. (2004) with respect to pore microstructure in the region. Then we analyzed seismic velocity tomography data to understand whether the deep extension of the Nagamachi-Rifu fault exposes the properties of a system with equilibrium geometry, using Takei (2002) method. Afterwards we applied the fractal model for elastic and electrical properties of porous rock to analyze of the results of collocated MT and velocity tomography experiments to specify the fine distribution of micropore connectivity in the region.

\section{Experimental Data}

Figure 1 shows the location of the observation points of the MT survey and the surface trace of the Nagamachi-Rifu fault. A star indicates the epicenter of an earthquake with 
(a)

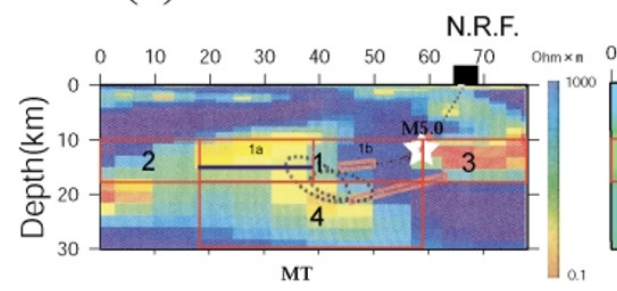

(b)

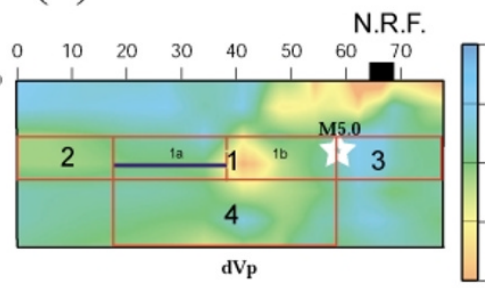

(c)

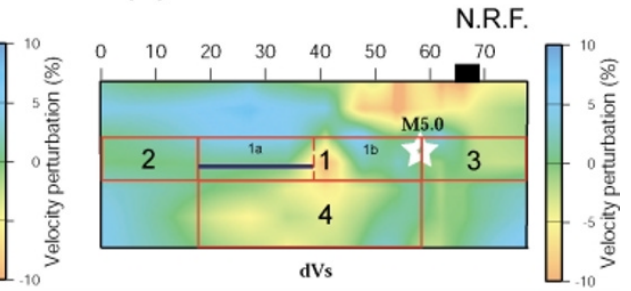

Fig. 2. (a) Electrical resistivity (after Ogawa et al., 2004), (b) $P$ wave, and (c) $S$ wave velocities variation (Nakajima, 2002) across the Nagamachi-Rifu fault. The surface location of the Nagamachi-Rifu fault is marked with the black rectangle and the N.R.F. abbreviation. The epicenter of an earthquake with M 5.0 is indicated by a star. The region 1 located at the depth 10-17 km and at the distance $0-40 \mathrm{~km}$ to the northwest from the M 5.0 hypocenter is referred in the present article as the deep extension of the Nagamachi-Rifu fault. The blue line indicates the slipping part of the deep extension of the Nagamachi-Rifu fault suggested by Nishimura et al. (2004). Region 1 was divided into region $1 \mathrm{a}$ and $1 \mathrm{~b}$ for the joint analysis of $V_{p}, V_{s}$ and $r$ data. Regions 2, 3 and 4 are the northwestern, southeastern and lower adjacent parts, respectively.

M 5.0, which occurred on this fault on 15 September 1998 at the depth of about $12 \mathrm{~km}$. The $P$ and $S$ wave velocity structures were obtained within the rectangle area in Fig. 1 with horizontal resolution of 5-7 km and vertical resolution of $5 \mathrm{~km}$ throughout the depth 0-30 km (Nakajima, 2002; Nakajima et al., 2004). The seismic velocities outside of the dense grid area were obtained for horizontal grid intervals of $20 \mathrm{~km}$. Wide-band magnetotelluric data were collected at 18 sites along the profile perpendicular to the NagamachiRifu fault trace (Ogawa et al., 2004).

Electrical resistivity obtained by Ogawa et al. (2004) is presented in Fig. 2(a). Results of 3D inversion of seismic velocity data, which are obtained and interpolated to 1 $\mathrm{km} \times 1 \mathrm{~km}$ grid along the MT profile by Nakajima (2002) and Nakajima et al. (2004), are shown in Fig. 2(b) and (c). The data are presented on the same scale. Several zones of low velocity and low resistivity are revealed along the profile. In the present paper, we concentrate on the region of the suggested deep extension of the NagamachiRifu fault (e.g. Sato et al., 2002). We analyze a region of $40 \mathrm{~km}$ length from the hypocenter of the M5.0 earthquake to the northwest along the MT survey line through the depths $10-17 \mathrm{~km}$ (region 1 in Fig. 2) as a main candidate for the deep extension of the Nagamachi-Rifu fault. Nishimura et al. (2004) suggested aseismic sliding within this region on a horizontal detachment fault connecting to the Nagamachi-Rifu fault at a depth of about $15 \mathrm{~km}$ (blue line in Fig. 2). The region includes the $10 \%$ low $V_{p}$ and $V_{s}$ velocity zone with the center located nearly $15 \mathrm{~km}$ from the hypocenter of the M5.0 earthquake at the depth of about 15 $\mathrm{km}$ and adjacent conductive zone of tens of $\mathrm{ohm} \cdot \mathrm{m}$. The low velocity anomaly of the suggested deep extension of the Nagamachi-Rifu fault exhibits moderate $V_{p} / V_{s}$ values of about 1.75 (Nakajima, 2002; Nakajima et al., 2004). The low $V_{p}$ and low $V_{s}$ anomaly was explained with existence of $\mathrm{H}_{2} \mathrm{O}$-filled pores with the relatively large aspect ratios of 0.05-0.1 (Nakajima, 2002; Nakajima et al., 2004). The low electrical resistivity zone to the west from the low velocity anomaly was also explained with the existence of fluid by Ogawa et al. (2004). It should be noted that the locations of low velocity and low resistivity anomalies are different, even though both the anomalies are in the region 1 . Thus, we divide the region 1 into two parts, namely region 1a and $1 \mathrm{~b}$, for the joint analysis of seismic velocities and resistiv- ity in chapter 4 . We also analyze the regions adjacent to the suggested area of the deep extension of the Nagamachi-Rifu fault, namely, northwestern, southeastern and lower regions (marked as 2, 3, and 4 in Fig. 2) for comparison with the region 1 .

\section{Pore Aspect Ratio and Porosity Estimation from Seismic Velocity Tomography Data}

Recently, several studies have pointed out the importance of $d \ln V_{s} / d \ln V_{p}$ as a proxy of the microstructure of a seismic anomaly (e.g., Kennett et al., 1998; Masters et al., 2000; Takei, 2002). The ratio $d \ln V_{s} / d \ln V_{p}$ can be determined from $V_{p}$ and $V_{s}$ data obtained at a number of grid points in an analyzed region without a priori suggestion of intact rock seismic velocities $V_{p 0}$ and $V_{s 0}$. Takei (2002) showed that $d \ln V_{s} / d \ln V_{p}$ is independent of $\phi$ for a small velocity variation case. Thus, if we assume that $\alpha$ is constant and the seismic velocity variations are only caused with porosity variation in the region, the value $d \ln V_{s} / d \ln V_{p}$ can be used for determining $\alpha$ from Takei's (2002) result. Then, porosity variation in the region can be also obtained from the velocity variation after the estimate of $\alpha$ using figure 5 of Takei (2002). Porosity variation here means a difference between the maximal and the minimal porosities in the analyzed region.

Takei (2002) has shown that for water-rock system at the depth $0-30 \mathrm{~km}$ in a region with the equilibrium geometry, the ratio $d \ln V_{s} / d \ln V_{p}$ takes values of $0.8-1.1$ corresponding to the $\alpha$ of $0.15-0.5$ and a region with nonequilibrium geometry demonstrates the larger values of the ratio $d \ln V_{s} / d \ln V_{p}$ such as 1.1-2.2 corresponding to $\alpha$ less then 0.15 . The occurrence histograms of $\ln V_{s}$ vs. $\ln V_{p}$ are presented in Fig. 3(a), (b), (c) and (d) for the deep extension of the Nagamachi-Rifu fault, northwestern, southeastern and lower part of the MT survey line, corresponding to region 1, 2, 3 and 4 in Fig. 2. The grid of the histogram is limited by the velocity data error of $0.2 \mathrm{~km} / \mathrm{s}$, which was determined using the root mean square of the arrival time residuals that are reported to be equal to $0.13 \mathrm{~s}$ and $0.28 \mathrm{~s}$ for $P$ and $S$ wave respectively (Nakajima, 2002; Nakajima et al., 2004). The colored lines in Fig. 3(a), (b) and (c)) show the $d \ln V_{s} / d \ln V_{p}$ values along the horizontal lines at depths of 11 (red), 14 (green) and $17 \mathrm{~km}$ (blue). The $d \ln V_{s} / d \ln V_{p}$ values along horizontal lines at depths of 21, 
(a)
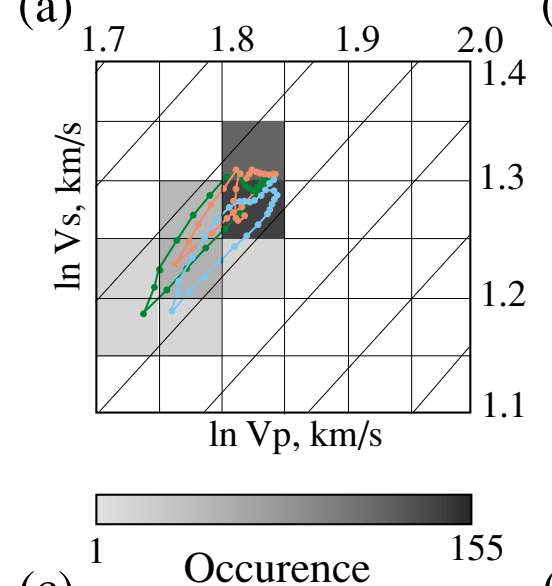

(c)

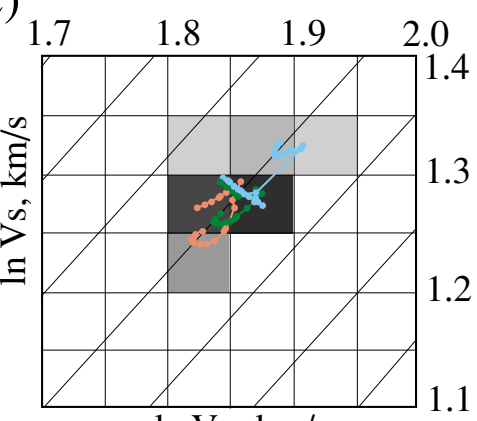

$\ln \mathrm{Vp}, \mathrm{km} / \mathrm{s}$

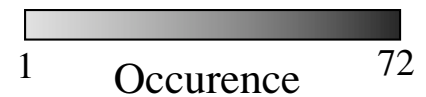

(b)

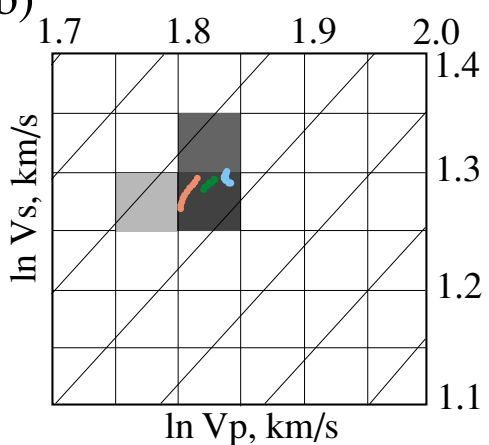

(d)
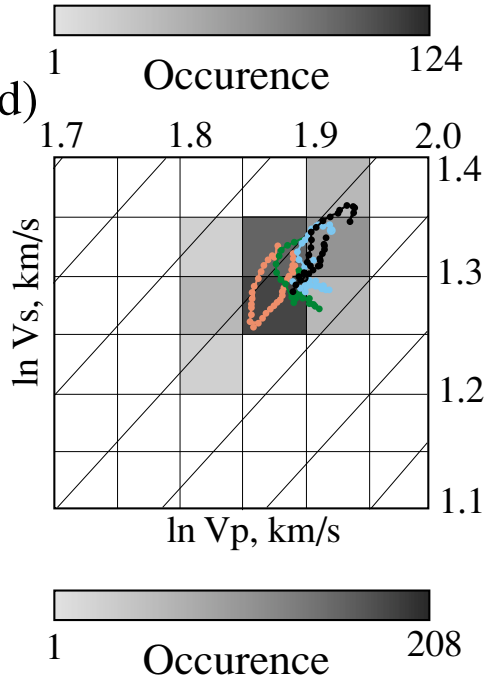

(d)

Fig. 3. Occurrence histogram of $\ln V_{s}$ vs. $\ln V_{p}$. (a) For the deep extension of the Nagamachi-Rifu fault, the region 1 in Fig. 2. The colored lines show the $d \ln V_{s} / d \ln V_{p}$ values along the horizontal lines at depths of 11 (red), 14 (green) and $17 \mathrm{~km}$ (blue). (b) For the northwest part of the cross-section, the region 2 in Fig. 2. (c) For the southeast part of the cross-section, the region 3 in Fig. 2. In (b) and (c) the colored lines have the same meanings as in (a). (d) For the lower part of the cross-section, the region 4 in Fig. 2. The colored lines show the $d \ln V_{s} / d \ln V_{p}$ values along the horizontal lines at depths of 21 (red), 24 (green), $27 \mathrm{~km}$ (blue) and $30 \mathrm{~km}$ (black). The tangent of the angle between the inclined straight lines and the horizontal axis is equal to 1.1 .

24, 27 and $30 \mathrm{~km}$ (red, green, cyan and black color respectively) in the region 4 are shown in Fig. 3(d). The tangent of the angle between the inclined straight lines and the horizontal axis is equal to 1.1 . We can estimate $\alpha$ and porosity variation in the regions as follows.

For the region 1 , the values $d \ln V_{s} / d \ln V_{p}$ for individual horizontal lines from 10 to $17 \mathrm{~km}$ can be seen to have a range from 1.2 to 2.2 (Fig. 3(a)). Thus, $\alpha$ in the region is estimated to be ranged $0.08-0.001$ on the base of the study of effect of pore geometry on $d \ln V_{s} / d \ln V_{p}$ (Takei, 2002). It should be noted that such values reveal the regions with the non-equilibrium pore geometry. Porosity variation in the region is, then, estimated from $\alpha$. The estimated porosity variation in the region 1 is ranged from 0.07 to $5 \%$. The range of the porosity variation is related to the uncertainty of the estimated $\alpha$. The values of the $d \ln V_{s} / d \ln V_{p}$ for both the regions 2 and 3 (Fig. 3(b) and (c) respectively), which are shown for horizontal lines 11, 14 and $17 \mathrm{~km}$ (red, green and cyan colors respectively) are within the errors of $V_{p}$ and $V_{s}$ data. Occurrence histograms for these regions also do not allow making a reliable conclusion about microstructure in the regions. For the region 4 the values $d \ln V_{s} / d \ln V_{p}$ for individual horizontal lines are within the limits of 1.2 to 2.2 in Fig. 3(d). The aspect ratio is estimated to be $0.08-0.001$, which is the same as in the region 1 . Thus, $\alpha$ in the region 4 also show the non-equilibrium pore geometry. The porosity variation is equal to $4.4 \%$ for $\alpha=0.08$ and $0.06 \%$ for $\alpha=0.001$ in the region 4 .

As stated in chapter 2, location of low resitivity, namely, region $1 \mathrm{a}$ and low velocity anomaly, namely, region $1 \mathrm{~b}$ is different in the region 1. It might be meaningful to compare the porosity variation in the low resistive anomaly and that in the other part of the region 1. The region 1(a) reveals porosity variation of $0.03 \%$ for the case of $\alpha=0.001$ and $2.5 \%$ for the case of $\alpha=0.08$. The porosity variation in the region $1 \mathrm{~b}$ is about twice of that in the region $1 \mathrm{a}$, coincides with the value estimated for the whole region 1.

On the basis of $V_{p}$ and $V_{s}$ analysis, the deep extension of the Nagamachi-Rifu fault, namely the region 1 , seems to be affected by deformation that preventing the fluid filled pores 
from achieving the equilibrium geometry. The region 4 under the deep extension of the Nagamachi-Rifu fault reveals similar properties. The accuracy of the seismic velocity tomography data in the regions 2 and 3 to the northwest and southeast from the deep extension of the Nagamachi-Rifu fault is insufficient to make reliable suggestion about the pore microstructure in the regions. Porosity variation is estimated to be equal to $0.07-5 \%$ for region 1 and $0.06-4.4 \%$ for region 4 . It should be noticed that the porosity variation in the region 1a estimated as $0.03-2.5 \%$ is noticeably smaller that the maximal porosity in the region $1 \mathrm{~b}$.

\section{Joint Analysis of Seismic Velocity and Resistiv- ity}

In the previous chapter, porosity and pore aspect ratio are estimated in the region of the deep extension of the Nagamachi-Rifu fault using only $P$ and $S$ wave velocity data. Electrical resistivity data obtained by the collocated MT experiment can hardly confirm or improve the values of the porosity and the aspect ratio estimated using the seismic velocity tomography data, because not porosity but pore connectivity and fluid resistivity drastically affect the effective resistivity. According to Hyndman and Shearer (1989), the change of a fluid resistivity of a factor of 2 leads to an error of $20-30 \%$ in the estimated porosity. For rock with $1 \%$ of aqueous inclusions, the resistivity may differ in more than 3 orders between the rock with isolated inclusions and that with film channels along grain edges (Hyndman and Shearer, 1989). Thus, electrical resistivity fails to give a reliable estimation of porosity. However, electrical resistivity is extremely sensitive to pore connectivity and can be valuable for imaging of fault zones (Jones, 1998). To estimate the connectivity in the regions 1 and 4 , which are shown to reveal non-equilibrium pore geometry, the experimental data of seismic velocities and electrical resistivity are compared with the simulated velocity-resistivity relationships for various pore microstructures.

A fractal model of elastic and electrical properties of porous rock (Pervukhina et al., 2003, 2004) is used for simulation of seismic velocities and electrical resistivity in the region of the deep extension of the Nagamachi-Rifu fault. Here we briefly describe the model and the model parameters that are chosen for simulation (see Appendix for technical details). The fractal model describes porous rock microstructure with the 3 elements, namely, liquid filled pore (element 1), rock matrix (element 2) and contact region (element 3) (Fig. 4(a) and (b)). A basic geometrical model to describe the geometry in Fig. 4(b) is schematically represented in Fig. 4(c); hereafter we will refer it as a partially interconnected pore model.

Two types of elementary units of cuboids of free eligible sizes: $l_{1}, l_{2}$, and $l_{3}$ (Fig. $4(\mathrm{~d})$ and (e)) are used to construct the partially interconnected pore model. A unit with isolated pore geometry, namely a unit A, describes an isolated pore surrounded by matrix material (Fig. 4(d)). A unit with interconnected pore geometry, namely a unit B, represents a matrix grain surrounded by pore space (Fig. 4(e)). Dimensions $a_{1}, a_{2}$ and $a_{3}$ describe thickness of matrix walls for the unit A or thickness of pore channels for the unit B. Aspect ratio of a pore is only defined for the unit A as $\left(l_{k}-a_{k}\right) /\left(l_{i}-a_{i}\right)$, where $l_{i}-a_{i}=l_{j}-a_{j} \neq l_{k}-a_{k}$. For the units $\mathrm{A}$ and $\mathrm{B}$, the regions marked with a numeral 3 , are defined as the contact regions in Fig. 4 (d) and (e). The contact region possibly has a fractal structure, i.e. filled with one or more subgenerations. For instance, in the partially interconnected pore model of Fig. 4(c), the zero-generation is represented by the unit A and its contact region is filled with the 1st-generation of units B.

Total pore volume of the model is equal to $V_{p}^{\text {total }}=$ $V_{p}^{\text {is }}+V_{p}^{\text {in }}$, where $V_{p}^{\text {is }}$ is pore volume of the total number of the units $\mathrm{A}$ and $V_{p}^{\text {in }}$ is pore volume of the total number of the units B. In the case of partially interconnected model presented in Fig. 4(c), $V_{p}^{\text {is }}=\prod_{i=1}^{3}\left(l_{i}^{0}-a_{i}^{0}\right)$ is the pore volume of zero-generation and $V_{p}^{\text {in }}=l_{1}^{1} a_{2}^{1} a_{3}^{1}+\left(l_{2}^{1}-a_{2}^{1}\right) a_{1}^{1} a_{3}^{1}+\left(l_{3}^{1}-\right.$ $\left.a_{3}^{1}\right) a_{1}^{1} a_{2}^{1}$ is the pore volume of the 1 st-generation. Hereafter, upper superscripts of geometrical sizes indicate generation numbers. The total porosity $\phi_{p}^{\text {total }}$ is equal to $V_{p}^{\text {total }} / V^{\text {total }}$, where $V^{\text {total }}=\prod_{i=1}^{3} l_{i}^{0}$ is the bulk volume of the model. The total porosity of the model with several generations of units $\mathrm{A}$ and $\mathrm{B}$ can be defined as $\phi_{p}^{\text {total }}=\phi_{p}^{\text {is }}+\phi_{p}^{\text {in }}$, where $\phi_{p}^{\text {in }}=V_{p}^{\text {in }} / V^{\text {total }}$ and $\phi_{p}^{\text {is }}=V_{p}^{\text {is }} / V^{\text {total }}$. For quantitative discussion of connectivity, we defined the parameter $C=V_{p}^{\text {in }} / V_{p}^{\text {total }}$ as the ratio of the pore volume of the total number of units B to the total pore volume. The parameter $C$ reflects connectivity of an isotropic model and ranges from 0 to 1: $C=0$ for a model with all the generations of units $\mathrm{A}\left(V_{p}^{\text {is }}=V_{p}^{\text {total }}, V_{p}^{\text {in }}=0\right)$ and $C=1$ for a model with all the generations of units $\mathrm{B}\left(V_{p}^{\text {in }}=V_{p}^{\text {total }}, V_{p}^{\text {is }}=0\right)$. A model with zero-generation of unit B (Fig. 4(e)) and without subgenerations in the contact region and $a_{1}^{0}=a_{2}^{0}=a_{3}^{0}$ will be mentioned as an isotropic tube model, for the following discussions.

Calculation of electrical and elastic properties is an iterative process for the model with one or more subgenerations in the contact region. First, the elastic moduli and resistivity are calculated for the smallest generation. The geometrical unit of the smallest generation is subdivided into serial or parallel connections of matrix, pore and contact region elements; and the resultant physical properties are calculated for those equivalent networks (see Appendix for details). Then, physical properties for the contact region of the upper generation are assumed to be equal to the physical properties of the previous generation. The process continues until the physical properties of the largest generation are calculated.

The velocity-resistivity curves in Fig. 5 are calculated for various microstructures, such as the isotropic tube model of one generation of the unit B and the partially interconnected pore model of 4 generations. The partially interconnected pore models are with zero-, 1st-, and 2nd-generations of unit A and 3rd- generation of units B. Pores of the units A have shapes of flat parallelepipeds with aspect ratio 0.08 , which is the upper limit of plausible aspect ratio obtained from seismic velocity analysis in chapter 3 . In the zero-, 1st-, and 2nd- generations, the pores are oriented in 3 perpendicular directions to make the model isotropic. The calculations with larger number of generations, namely, 6 generations of unit A showed that the resistivity-velocity relationships do not display essential difference for the par- 
(a)
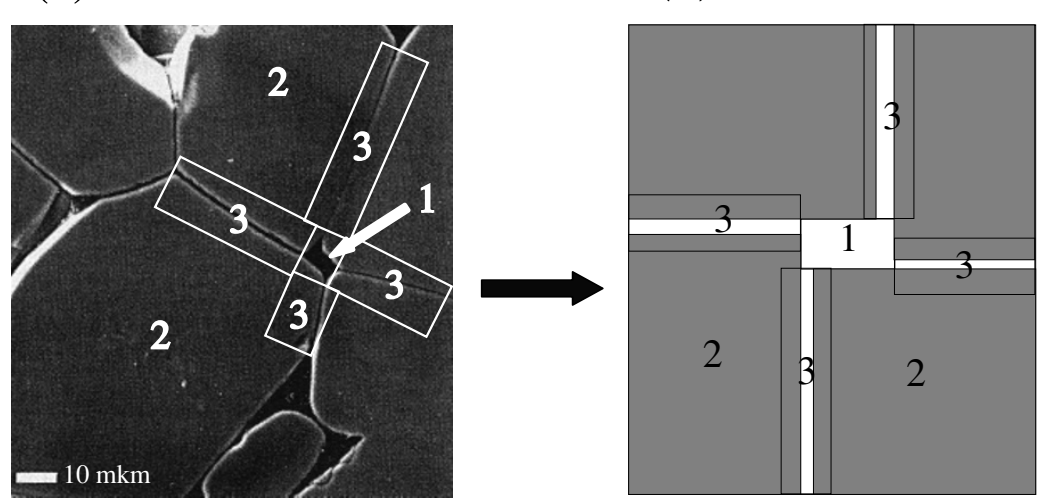

$1 \square$ Pore

$2 \square$ Matrix

$3 \rightleftharpoons$ Contact region (b)

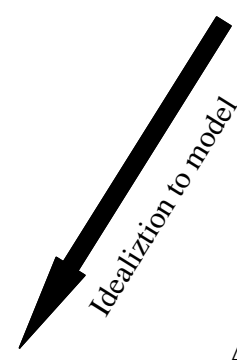

(d)

Unit A with isolated pore geometry

$1_{1}$

(c)

Partially interconnected pore model

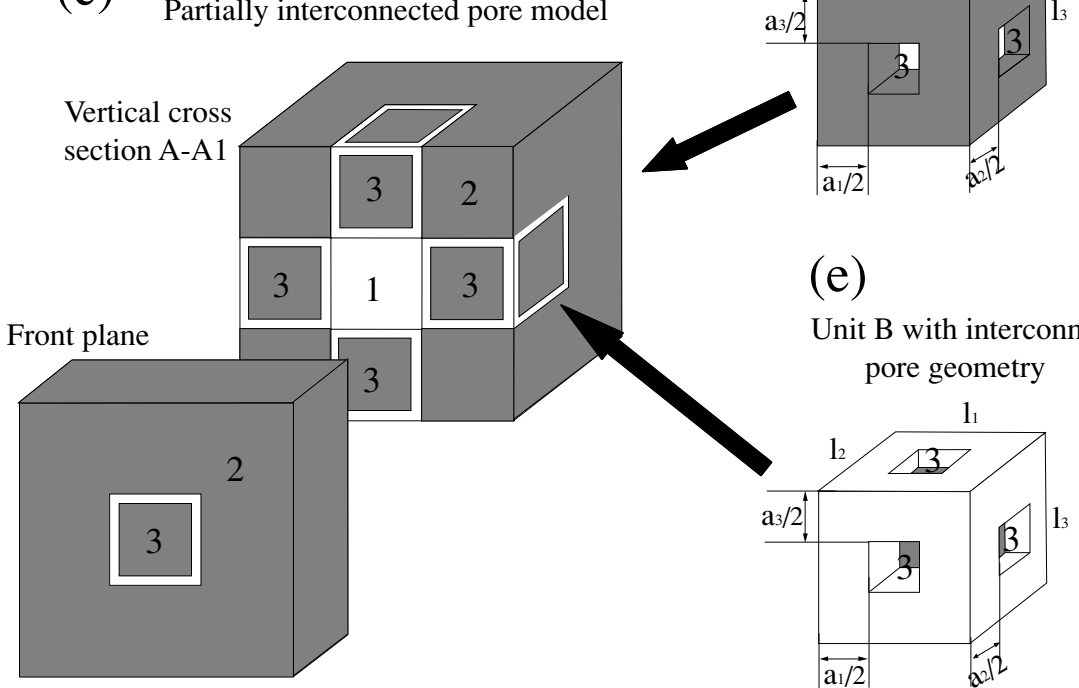

Fig. 4. (a) Photograph of sectioned and polished surface of quartzite synthesized in presence of saline water (after Watson and Brenan, 1987). Three basic elements for the fractal model of rock microstructure are indicated as: 1-liquid filled pore, 2-rock matrix and 3-contact region. (b) Simplified pore rock microstructure from Fig. 4(a). (c) The partially interconnected pore model for representing the simplified microstructure of (b). (d) Unit A with isolated pore geometry. The unit A describes isolated pore surrounded by matrix material. Free eligible dimensions $l_{1}, l_{2}$ and $l_{3}$ describe 3D sizes of grains; $a_{1}, a_{2}$ and $a_{3}$ describe thickness of matrix walls. (e) Unit B with interconnected pore geometry. The unit B presents matrix surrounded by pore filling. Dimensions $l_{1}, l_{2}$ and $l_{3}$ have the same meanings as in (d); $a_{1}, a_{2}$ and $a_{3}$ thickness of pore channels.

tially interconnected model up to $10 \%$ of the velocity variations. The following values are adopted as the parameters for simulating. Resistivity of the intact rock $r_{m}$ is chosen as $1.5 \cdot 10^{4} \Omega \cdot \mathrm{m}$ that is the maximal measured resistivity along the MT profile. Fluid resistivity $r_{p}$ is adopted as $0.02((\mathrm{~m}$ (Simpson and Warner, 1998) that seems to be a justifiable lower limit to use in calculating a maximal resistivity variation corresponding to velocity variation caused with porosity or geometry alteration. Water sound speed is chosen as $2.07 \mathrm{~km} / \mathrm{s}$ that corresponds to the pressure of $0.4 \mathrm{GPa}$ and the temperature of $200^{\circ} \mathrm{C}$ (Clark, 1966). Density of matrix rock is assumed to be equal to the maximal density of granite $2809 \mathrm{~kg} / \mathrm{m}^{3}$ (Clark, 1966). Density of pore-filling fluid is taken to be $1100 \mathrm{~kg} / \mathrm{m}^{3}$ that corresponds to the pressure of $0.4 \mathrm{GPa}$ and the temperature of $200^{\circ} \mathrm{C}$ (Ahrens, 2000). Since $V_{p 0}$ and $V_{s 0}$ values are difficult to be assumed, we fulfill simulations with various values of $V_{p 0}$ and $V_{s 0}$ and find that up to $5 \%$ increasing of $V_{p 0}$ and $V_{s 0}$ from the maximal velocities in regions 1 and 4 measured by Nakajima (2002) and Nakajima et al. (2004) does not noticeably af- 
(a)

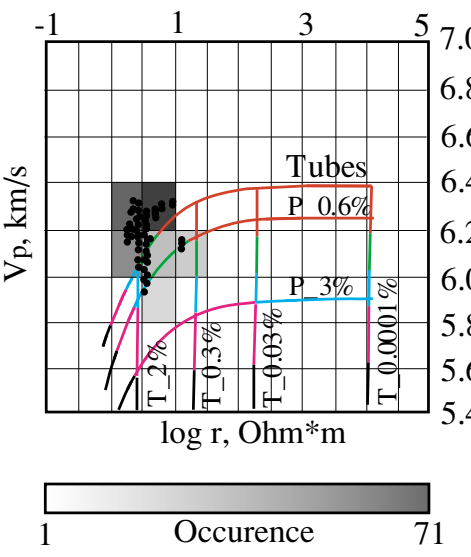

(b)

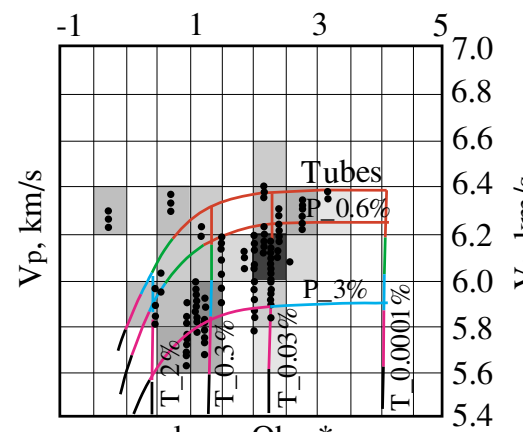

$\log \mathrm{r}, \mathrm{Ohm}^{*} \mathrm{~m}$

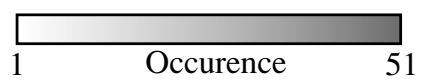

(c)
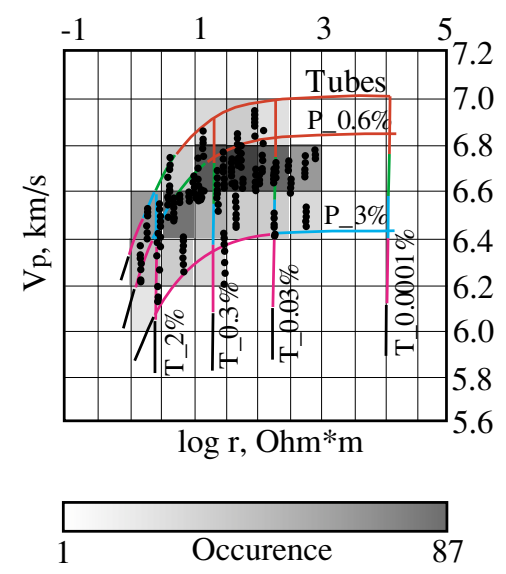
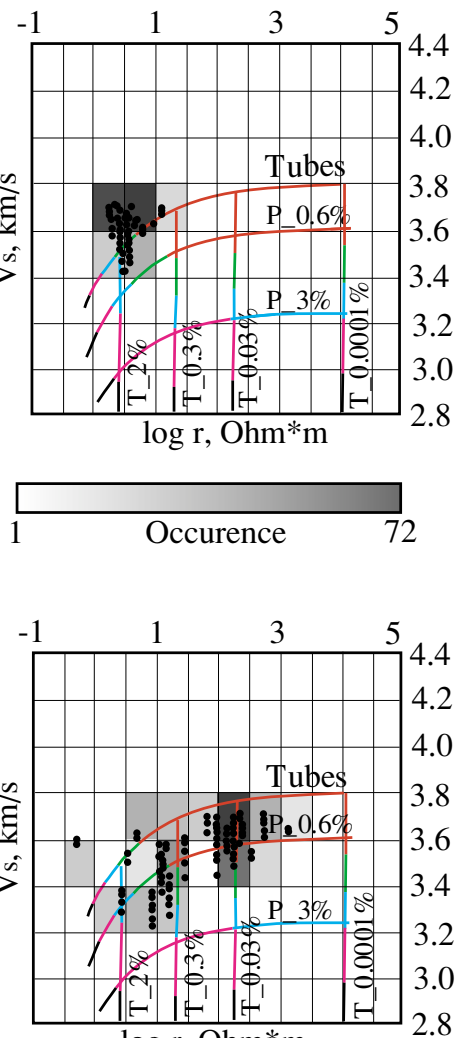

$\log \mathrm{r}, \mathrm{Ohm} * \mathrm{~m}$
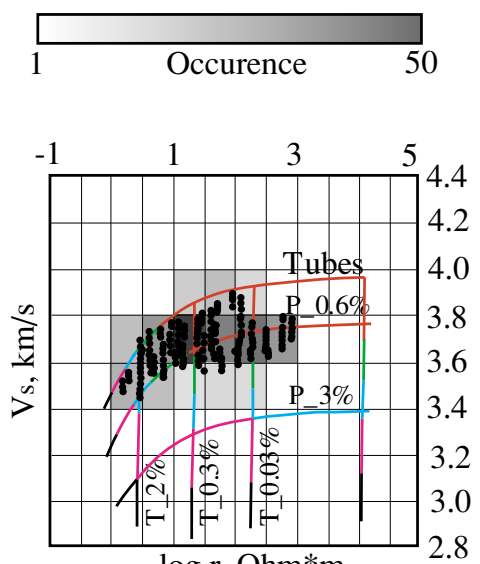

$\log \mathrm{r}, \mathrm{Ohm} * \mathrm{~m}$

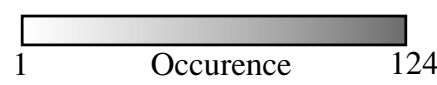

Fig. 5. $V_{p}-r$ and $V_{s}-r$ occurrence histograms for the deep extension of the Nagamachi-Rifu fault: (a) for the region 1a, (b) for the region 1b, and (c) for the region 4 . The observed data are shown by solid dots. The resistivity against porosity curves are presented for the isotropic tube model and partially interconnected pore models with the aspect ratio of 0.08 . The velocity-resistivity curves marked with $\mathrm{T}$ letter are calculated with the fixed porosity $\phi_{p}^{\int}$ of $0.0001 \%, 0.03 \%, 0.3 \%$ and $2 \%$ (the porosity is written along the curves). The $P$ marked velocity-resistivity curves are calculated with the fixed porosity $\phi_{p}^{\text {is }}$ of $0.6 \%$ and $3 \%$. The total porosity is indicated with the color of the curves: red indicates the porosity less than $1 \%$, green does less than $2 \%$, light blue shows the total porosity less than $3 \%$, magenta does less than $5 \%$, black does less than $7 \%$.

fect the obtained values of connectivity in the regions. To exemplify following calculations, the intact rock velocities are assumed to be equal to $V_{p 0}=6.39 \mathrm{~km} / \mathrm{s}$ and $V_{s 0}=3.80$ $\mathrm{km} / \mathrm{s}$ and $V_{p 0}=7.02 \mathrm{~km} / \mathrm{s}$ and $V_{s 0}=3.96 \mathrm{~km} / \mathrm{s}$ for region 1 and 4 , respectively, which are $1 \%$ higher values than the maximal measured velocities in each region.

For the joint velocity-resitivity analysis, the region 1 is divided into two parts, namely, region 1a and $1 \mathrm{~b}$ (Fig. 2). $V_{p}-r$ and $V_{s}-r$ frequency histograms for regions $1 \mathrm{a}, 1 \mathrm{~b}$ and
4 are presented in Fig. 5(a), (b) and (c), respectively. The observed velocities against resistivities for individual grid nodes are shown by solid dots. The velocity-resistivity curves marked with $\mathrm{T}$ letter are calculated with the fixed $\phi_{p}^{\text {in }}$ of $0.0001 \%, 0.03 \%, 0.3 \%$ and $2 \%$; these values are written along the T-marked curves. The total porosity $\phi_{p}^{\text {total }}$ increases only due the increase of $\phi_{p}^{\text {is }}$. P-marked velocityresistivity curves are calculated with fixed $\phi_{p}^{\text {is }}$ of $0.6 \%$ and 

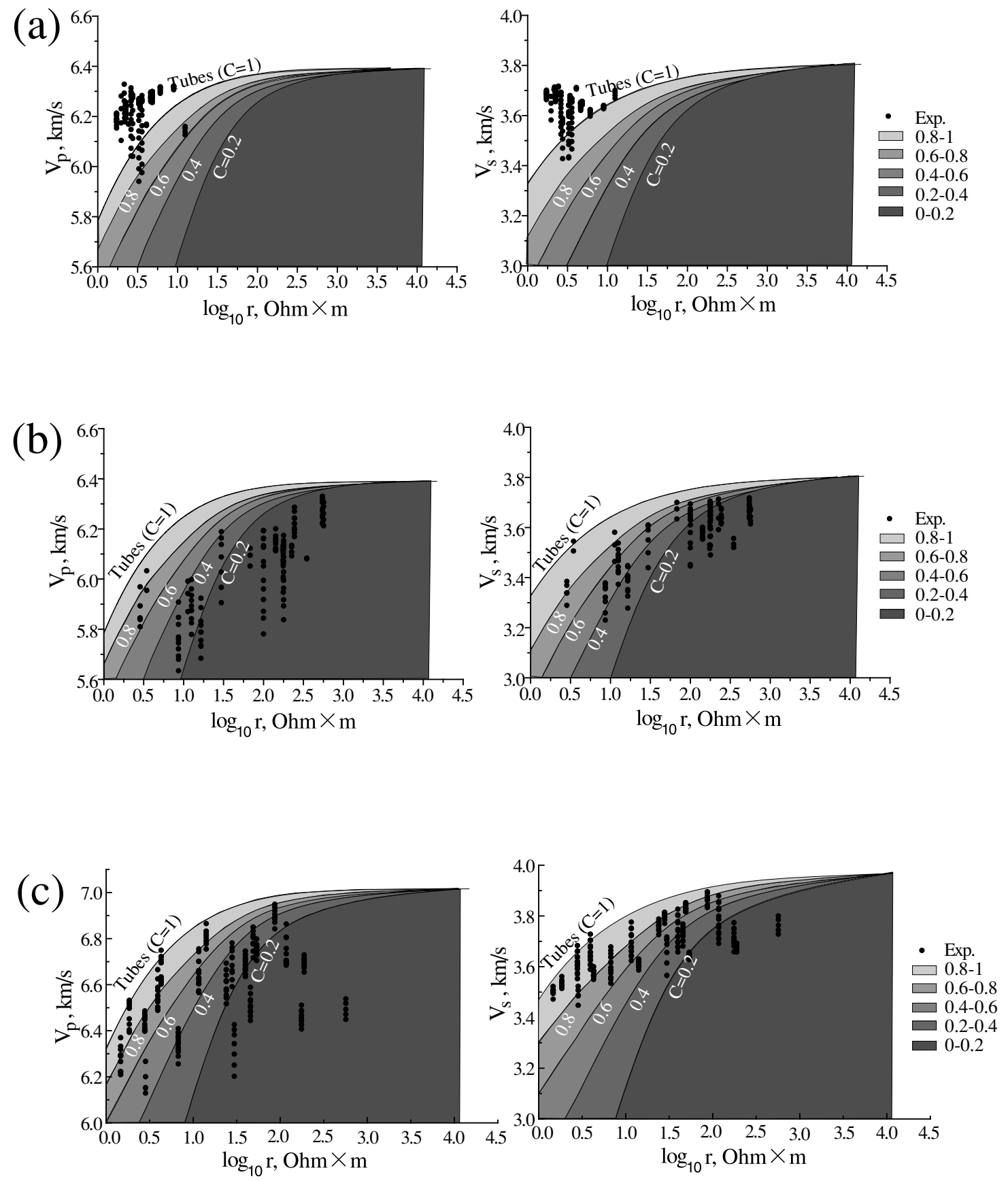

Fig. 6. Distribution of isotropic connectivity C value, the ratio of the pore volume $V_{\text {in }}^{p}$ of the total number of units B to the total pore volume $V_{p}^{\text {total }}$, in comparison with the experimental data: (a) for region $1 \mathrm{a}$, (b) for region $1 \mathrm{~b}$ and (c) for region 4 .

3\%; these values are indicated along the P-marked curves. The total porosity $\phi_{p}^{\text {total }}$ increase results from the increase of $\phi_{p}^{\text {in }}$. The total porosity $\phi_{p}^{\text {total }}$ is indicated with the color of the curves: red indicates the porosity less than $1 \%$, green does less than $2 \%$, light blue shows the total porosity less than 3\%, magenta does less than 5\%, black does less than $7 \%$ and simulated data for the total porosity more than $7 \%$ are omitted.

In the region 1a the experimental data reveal low resistivity and high velocity. Velocity-resistivity data in this region can be generally explained with porosity of $2-3 \%$ and tubetype micropore structure (Fig. 5(a)). However, some of the points with high velocity and low resistivity are rather above the theoretical curve for isotropic tubes. This is probably explained by some reasons: one is measuring uncertainty or uncertainty of $V_{p 0}$ and $V_{s 0}$ choices. The other is a possibility of anisotropic pore geometry which can result in lower resistivities than the resistivity-velocity curve for isotropic tubes. Anisotropic pore geometry is quite possible in regions with non-equilibrium state, but it cannot be proved on the basis on the existing experimental data and requires further investigations. The experimental points in both regions $1 \mathrm{~b}$ and 4 can be satisfactorily explained with partially interconnected pores with the total porosity $\phi_{p}^{\text {total }} \approx 0.01-5 \%$ with porosity of generation with isolated pore geometry $\phi_{p}^{\text {is }} \leq 3 \%$ (Fig. 5(b) and (c)). Figures 6(a), (b) and (c) 
Table 1. Results of the analysis of the $V_{p}, V_{s}$ and resistivity along the deep extension of the Nagamachi-Rifu fault.

\begin{tabular}{cccc}
\hline Region & Porosity variation, $\Delta \phi$ & Aspect ratio, $\alpha$ & $C=V_{p}^{\text {in }} / V_{p}^{\text {total }}$ \\
\hline 1a & $0.03-2.5 \%$ & $-*$ & 1. \\
1b & $0.07-5.0 \%$ & $0.08-0.001$ & $0 .-0.4$ \\
4 & $0.06-4.4 \%$ & $0.08-0.001$ & $0 .-1$. \\
\hline
\end{tabular}

$*^{*} \alpha$ cannot be defined for region $1 \mathrm{a}$, because all the pores are interconnected pores in the case of $C=1$, which are described with the unit B geometry and aspect ratio was not defined.

show the distribution of $C\left(=V_{p}^{\text {in }} / V_{p}^{\text {total }}\right)$ value in comparison with the experimental data for regions $1 \mathrm{a}, 1 \mathrm{~b}$ and 4 . The data in region 1a are explained with $C=1$. The majority of the experimental data in the region $1 \mathrm{~b}$ can be explained with $C<0.4$. The experimental data in region 4 reveal rather high range of connectivity, namely, $C$ values range from 0 to 1 .

All the results of the joint analysis of seismic velocities and electrical resistivity for regions $1 \mathrm{a}, 1 \mathrm{~b}$ and 4 are summarized in Table 1. The regions $1 \mathrm{~b}$ and 4 exhibit the $\alpha$ of $0.08-0.001$, suggesting non-equilibrium pore geometry in the regions. We note that $\alpha$ can not be defined for region 1a, because all the pores are interconnected pores in the case of $C=1$, which are described with the unit B geometry and aspect ratio was not defined. The higher porosity and lower porosity variations are found in the region $1 \mathrm{~b}$ and $1 \mathrm{a}$, respectively. In the region 4 the porosity variation is estimated to have intermediate values. The highest value of connectivity $(C=1)$ is observed in the region 1a. The lowest connectivity values of $0-0.4$ are found in region $1 \mathrm{~b}$. Connectivity in region 4 ranges in from 0 to 1 . Thus, the region 1a has low porosity variation and high connectivity in comparison with region $1 \mathrm{~b}$ and 4 . It should be again noted that region 1a corresponds to the suggested slip region by Nishimura et al. (2004). This may suggest that the high connectivity is a better indicator than the porosity variation is for a strongly deformed region.

\section{Summary}

The mutual analysis of resistivity and seismic velocity tomography data from the collocated seismic velocity tomography and MT experiments is demonstrated to be useful for revealing of the fault zones in mid and lower crust. The following method is used to investigate microstructure of the region of the suggested deep extension of the NagamachiRifu fault. We find the non-equilibrium pore microstructure in the deep extension of the Nagamachi-Rifu fault on the basis of the spatial variation of the $d \ln V_{s} / d \ln V_{p}$ analysis. Porosity and aspect ratio are estimated using Takei (2002) model. Then resistivity-velocity data are compared with the simulating results of the fractal model of elastic and electrical properties of porous rock. It is shown that the northwestern part of the deep extension of the Nagamachi-Rifu fault demonstrates the highest pore connectivity $(C=1)$. This implies that the most deformed region of the deep extension of the Nagamachi-Rifu fault is $20-40 \mathrm{~km}$ to the northwest from the hypocenter of M5.0 earthquake.

Acknowledgments. We are indebted to Dr. Y. Ogawa, Dr. N.
Umino and Dr. J. Nakajima for providing us magnetotelluric and seismic velocity tomography data used in this study. We also thank them for valuable comments during the study. We gratefully acknowledge Dr. Takei and an anonymous reviewer who have provided thoughtful reviews. We thank an editor of the issue Dr. Iio for his useful comments. This study is a part of the Comprehensive research program on flow and slip process in and below the seismogenic region sponsored by Ministry of Education, Culture, Sports, Science and Technology (MEXT). M. Pervukhina was supported by Japan Science and Technology Agency.

\section{Appendix.}

Detailed description of the fractal model of elastic and electrical properties of porous rock used in this study is given by Pervukhina et al. (2003, 2004). Here we give brief derivation of porosity, density and electrical resistivity for the models of units with isolated and interconnected pore geometry, obtained by Pervukhina et al. (2003, 2004). We give final formulas for elastic modules which were obtained by Spangenberg (1998) for a unit with interconnected pore geometry, namely the unit B, and were similarly derived for the unit with isolated pore geometry, namely the unit A, by Pervukhina et al. (2003, 2004).

Porosity of the model with $\mathrm{n}$ subgenerations in contact region can be calculated as

$$
\phi=\frac{V_{p}^{\text {total }}}{V^{\text {total }}}=\frac{V_{p}^{0}+\sum_{g=1}^{n} V_{p}^{g} N_{g}^{\text {total }}}{\prod_{i=1}^{3} l_{i}^{0}},
$$

where $V_{p}^{\text {total }}$ is the pore volume in the all $n$ generations, $V^{\text {total }}$ is bulk volume of the zero-generation $V_{b}^{0}=\prod_{i=1}^{3} l_{i}^{0}$, $V_{p}^{g}$ is the pore space in a unit of the g-generation and $N_{g}^{\text {total }}$ is the total number of units of $g$-generation in the model. Pore space of the g-generation for a unit $\mathrm{B}$ is equal to $V_{p}^{g}=l_{1}^{g} a_{2}^{g} a_{3}^{g}+\left(l_{2}^{g}-a_{2}^{g}\right) a_{1}^{g} a_{3}^{g}+\left(l_{3}^{g}-a_{3}^{g}\right) a_{1}^{g} a_{2}^{g}$ and for the unit $\mathrm{A}$ is equal to $V_{p}^{g}=\prod_{i=1}^{3}\left(l_{i}^{g}-a_{i}^{g}\right)$.

Number of units of $g$-generation is calculated for assumption that the units of $g$-generation are filled compactly the contact region of the upper ( $g$-1)-generation unit. Volume of contact region of the $(g-1)$-generation unit is equal to

$$
\begin{aligned}
V_{c}^{g-1} & =\sum_{i, j, k=1}^{3}\left(l_{i}^{g-1}-a_{i}^{g-1}\right)\left(l_{j}^{g-1}-a_{j}^{g-1}\right) a_{k}^{g-1} \overline{\delta_{i j k}} ; \overline{\delta_{i j k}} \\
& =\left\{\begin{array}{l}
1, \quad i \neq j \neq k \\
0, i=j, i=k, j=k
\end{array}\right.
\end{aligned}
$$

The number of units of $g$-generation in the contact region of $(g-1)$-generation can be calculated as the ratio of the volume 

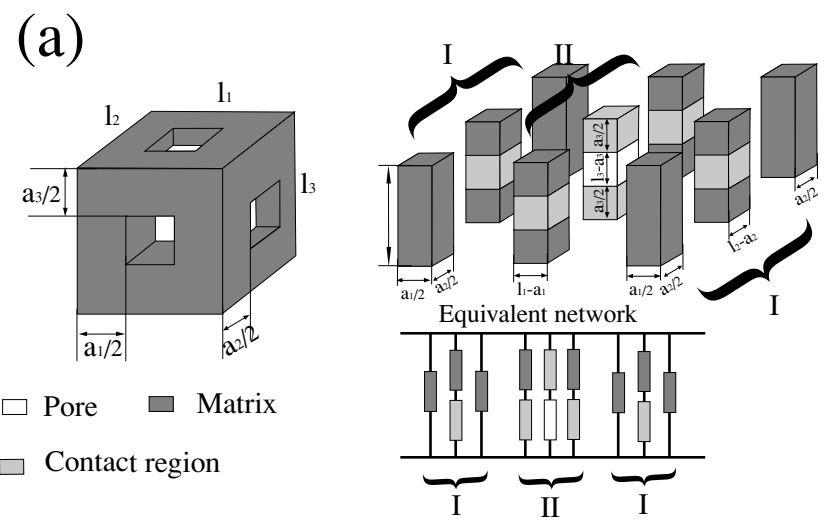

(b)
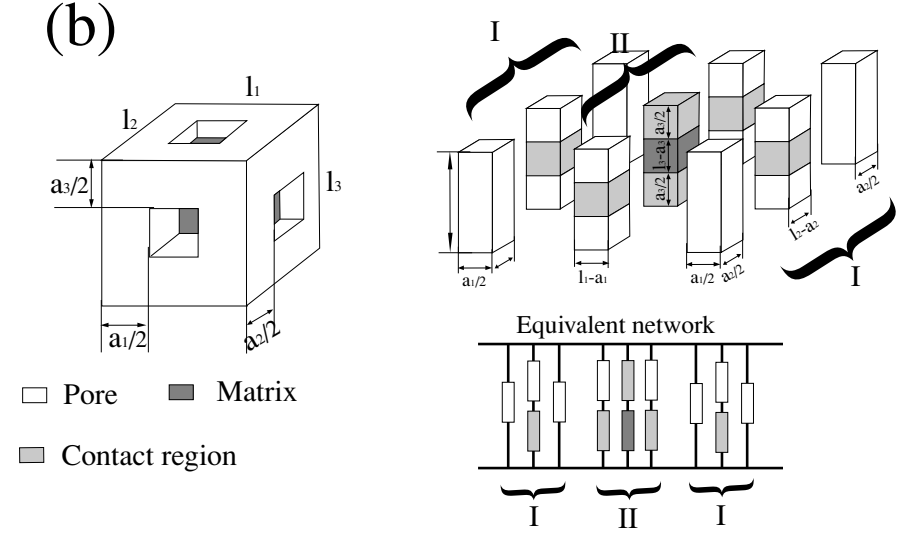

(c)

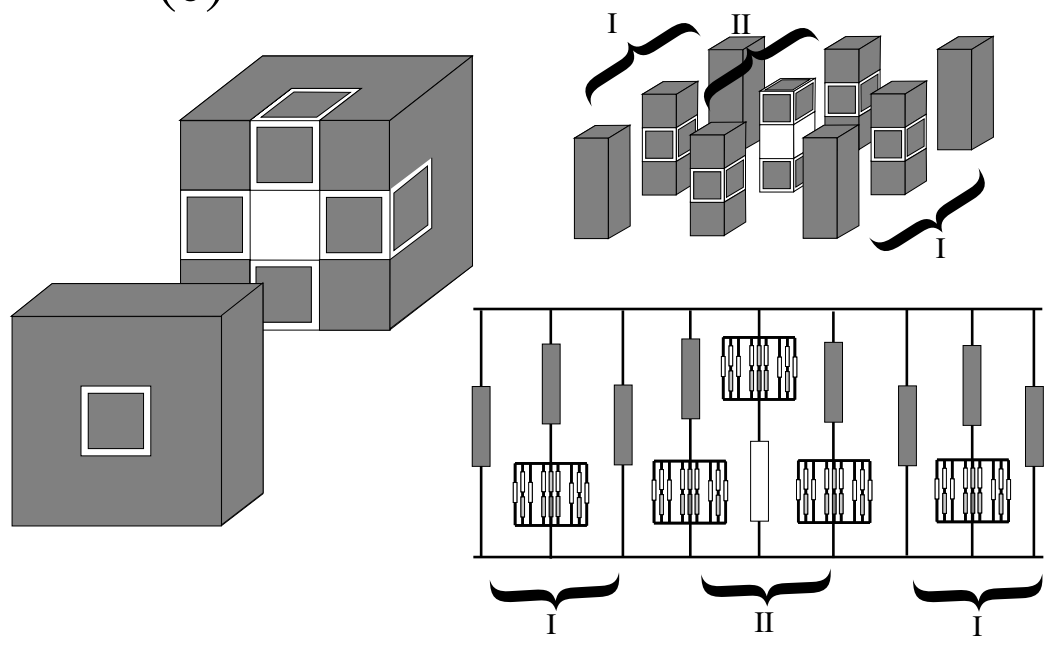

Fig. A.1. Equivalent network for (a) the unit A, (b) the unit Bl, and (c) the partially interconnected pore model.

of contact region of ( $g$-1)-generation to the bulk volume of The total number of the units of $g$-generation equals to $g$-generation

$$
N_{g}^{\text {total }}=\prod_{k=1}^{g} N_{k}
$$

$$
\begin{array}{r}
N_{g}=\frac{V_{c}^{g-1}}{V_{b}^{g}}=\frac{\sum_{i, j, k=1}^{3}\left(l_{i}^{g-1}-a_{i}^{g-1}\right)\left(l_{j}^{g-1}-a_{j}^{g-1}\right) a_{k}^{g-1} \overline{\delta_{i j k}}}{\prod_{i=1}^{3} l_{i}^{g}}, \\
\overline{\delta_{i j k}}=\left\{\begin{array}{l}
1, \quad i \neq j \neq k \\
0, i=j, i=k, j=k
\end{array}\right.
\end{array}
$$

Density for the model can be calculated as

$$
\rho=\frac{V_{p}^{\text {total }} \rho_{p}+V_{m}^{\text {total }} \rho_{m}+\left(\prod_{i=1}^{3} l_{i}^{0}-V_{m}^{\text {total }}-V_{p}^{\text {total }}\right) \rho_{c}}{\prod_{i=1}^{3} l_{i}^{0}}
$$


Table A.1. Resistance and elastic moduli for the vertical subdivision for the unit A and unit B (Fig. 4(d), (e)).

\begin{tabular}{|c|c|c|}
\hline & Unit A & Unit B \\
\hline \multirow[t]{4}{*}{$\mathrm{R}$} & $\begin{array}{l}R=\frac{R_{I} R_{I I}}{R_{I}+R_{I I}}, \\
\text { where }\end{array}$ & $\begin{array}{l}R=\frac{R_{I} R_{I I}}{R_{I}+R_{I I}}, \\
\text { where }\end{array}$ \\
\hline & $R_{I}=\frac{1}{a_{1}} \frac{r_{m} l_{3}\left(r_{m} a_{3}+r_{c}\left(l_{3}-a_{3}\right)\right)}{r_{m} l_{3}\left(l_{2}-a_{2}\right)+r_{m} a_{2} a_{3}+r_{c} a_{2}\left(l_{3}-a_{3}\right)}$ & $R_{I}=\frac{1}{a_{1}} \frac{r_{p} l_{3}\left(r_{p} a_{3}+r_{c}\left(l_{3}-a_{3}\right)\right)}{r_{p} l_{3}\left(l_{2}-a_{2}\right)+r_{p} a_{2} a_{3}+r_{c} a_{2}\left(l_{3}-a_{3}\right)}$ \\
\hline & and & and \\
\hline & $R_{I I}=\frac{1}{\left(l_{1}-a_{1}\right)} \frac{\left(r_{m} a_{3}+r_{c}\left(l_{3}-a_{3}\right)\right)\left(r_{c} a_{3}+r_{p}\left(l_{3}-a_{3}\right)\right)}{\left(r_{c} a_{3}+r_{p}\left(l_{3}-a_{3}\right)\right) a_{2}+\left(r_{m} a_{3}+r_{c}\left(l_{3}-a_{3}\right)\right)\left(l_{2}-a_{2}\right)}$ & $R_{I I}=\frac{1}{\left(l_{1}-a_{1}\right)} \frac{\left(r_{p} a_{3}+r_{c}\left(l_{3}-a_{3}\right)\right)\left(r_{c} a_{3}+r_{m}\left(l_{3}-a_{3}\right)\right)}{\left(r_{c} a_{3}+r_{m}\left(l_{3}-a_{3}\right)\right) a_{2}+\left(r_{p} a_{3}+r_{c}\left(l_{3}-a_{3}\right)\right)\left(l_{2}-a_{2}\right)}$ \\
\hline M & $M_{i}=\frac{a_{j} a_{k} M_{m}}{l_{j} l_{k}}+\frac{l_{i} M_{m} M_{c}\left(\left(l_{j}-a_{j}\right) a_{k}+\left(l_{k}-a_{k}\right) a_{j}\right)}{l_{j} l_{k}\left(a_{i} M_{c}+\left(l_{i}-a_{i}\right) M_{m}\right)}+\frac{\left(l_{j}-a_{j}\right)\left(l_{k}-a_{k}\right) l_{i} M_{c} M_{p}}{l_{j} l_{k}\left(\left(l_{i}-a_{i}\right) M_{c}+a_{i} M_{p}\right)}$ & $M_{i}=\frac{a_{j} a_{k} M_{p}}{l_{j} l_{k}}+\frac{l_{i} M_{p} M_{c}\left(\left(l_{j}-a_{j}\right) a_{k}+\left(l_{k}-a_{k}\right) a_{j}\right)}{l_{j} l_{k}\left(a_{i} M_{c}+\left(l_{i}-a_{i}\right) M_{p}\right)}+\frac{\left(l_{j}-a_{j}\right)\left(l_{k}-a_{k}\right) l_{i} M_{c} M_{m}}{l_{j} l_{k}\left(\left(l_{i}-a_{i}\right) M_{c}+a_{i} M_{m}\right)}$ \\
\hline$\mu$ & $\mu_{i}=\frac{a_{j} a_{k} \mu_{m}}{l_{j} l_{k}}+\frac{l_{i} \mu_{m} \mu_{c}\left(\left(l_{j}-a_{j}\right) a_{k}+\left(l_{k}-a_{k}\right) a_{j}\right)}{\left.l_{j} l_{k} a_{i} \mu_{c}+\left(l_{i}-a_{i}\right) \mu_{m}\right)}$ & $\mu_{i}=\frac{\left(l_{j}-a_{j}\right)\left(l_{k}-a_{k}\right) l_{i} \mu_{c} \mu_{m}}{l_{j} l_{k}\left(\left(l_{i}-a_{i}\right) \mu_{c}+a_{i} \mu_{m}\right)}$ \\
\hline
\end{tabular}

where $V_{p}^{\text {total }}=V_{p}^{0}+\sum_{g=1}^{n}\left(V_{p}^{g} \prod_{k=1}^{g} N_{k}\right), V_{m}^{\text {total }}=V_{m}^{0}+$ $\sum_{g=1}^{n}\left(V_{m}^{g} \prod_{k=1}^{g} N_{k}\right)$ and $\rho_{c}$ is the density of the contact region that is equal to the density of the unit of the 1 stgeneration. Pore volume equals to $V_{p}^{g}=l_{1}^{g} a_{2}^{g} a_{3}^{g}+\left(l_{2}^{g}-\right.$ $\left.a_{2}^{g}\right) a_{1}^{g} a_{3}^{g}+\left(l_{3}^{g}-a_{3}^{g}\right) a_{1}^{g} a_{2}^{g}$ for $g$-generation of the units B and equals to $V_{p}^{g}=\prod_{i=1}^{3}\left(l_{i}^{g}-a_{i}^{g}\right)$ for $g$-generation of the units A. Matrix volume equals to $V_{m}^{g}=\prod_{i=1}^{3}\left(l_{i}^{g}-a_{i}^{g}\right)$ for $g$-generation of the units $\mathrm{B}$ and equals to $V_{m}^{g}=l_{1}^{g} a_{2}^{g} a_{3}^{g}+$ $\left(l_{2}^{g}-a_{2}^{g}\right) a_{1}^{g} a_{3}^{g}+\left(l_{3}^{g}-a_{3}^{g}\right) a_{1}^{g} a_{2}^{g}$ for $g$-generation of the units A. The calculation of the density of the model is iterative process and the $\rho_{c}$ for the smallest generation is assumed to be equal to $\rho_{m}$.

To calculate the resistivity of the smallest generation of either the unit A or unit B, the unit is divided into rectangular elements of pore, matrix and contact region with resistivities $r_{p}, r_{m}$, and $r_{c}$, respectively (Fig. A.1(a), (b)). Resistivity of contact region $r_{c}$ is assumed to be equal to resistivity of the matrix material $r_{m}$ for the smallest generation. The rectangular elements are combined into parallel circuits. This approximation holds true for the case that resistivity variation is small and the electric equipotential surfaces are almost perpendicular to the current direction. The resistances of the equivalent networks are calculated as follows. The elements with identical geometry and composition can be combined directly. For example, the resistance $R_{I}$ of the left and right braced elements in Fig. A.1(b) can be calculated as a harmonic average of resistance $R_{1}$ of vertical pore channels and the resistance $R_{2}$ of serial connection of horizontal pore channel with contact region:

$$
R_{I}=\frac{R_{1}^{I} R_{2}^{I}}{R_{1}^{I}+R_{2}^{I}}
$$

where

$$
R_{1}^{I}=\frac{r_{p} l_{3}}{a_{1} a_{2}}
$$

and

$$
R_{2}^{I}=\frac{r_{p} a_{3}}{a_{1}\left(l_{2}-a_{2}\right)}+\frac{r_{c}\left(l_{3}-a_{3}\right)}{a_{1}\left(l_{2}-a_{2}\right)} .
$$

Finally,

$$
R_{I}=\frac{1}{a_{1}} \frac{r_{p} l_{3}\left(r_{p} a_{3}+r_{c}\left(l_{3}-a_{3}\right)\right)}{r_{p} l_{3}\left(l_{2}-a_{2}\right)+r_{p} a_{2} a_{3}+r_{c} a_{2}\left(l_{3}-a_{3}\right)} .
$$

Correspondingly, the resistance of the braced middle part is equal to

$$
R_{I I}=\frac{R_{1}^{I I} R_{2}^{I I}}{R_{1}^{I I}+R_{2}^{I I}}
$$

where

$$
R_{1}^{I I}=\frac{r_{p} a_{3}+r_{c}\left(l_{3}-a_{3}\right)}{\left(l_{1}-a_{1}\right) a_{2}}
$$

and

$$
R_{2}^{I I}=\frac{r_{c} a_{3}+r_{m}\left(l_{3}-a_{3}\right)}{\left(l_{1}-a_{1}\right)\left(l_{2}-a_{2}\right)}
$$

Substitution of (A.6-A.7) to (A.5) gives

$$
\begin{aligned}
& R_{I I}=\frac{1}{\left(l_{1}-a_{1}\right)} \\
& \frac{\left(r_{p} a_{3}+r_{c}\left(l_{3}-a_{3}\right)\right)\left(r_{c} a_{3}+r_{m}\left(l_{3}-a_{3}\right)\right)}{\left(r_{c} a_{3}+r_{m}\left(l_{3}-a_{3}\right)\right) a_{2}+\left(r_{p} a_{3}+r_{c}\left(l_{3}-a_{3}\right)\right)\left(l_{2}-a_{2}\right)} .
\end{aligned}
$$

The resistance of the equivalent network can be found by the substitution of (A.4) and (A.8) to the formula for the resistance of the parallel connection of two elements with resistance $R_{I}$ (A.4) and $R_{I} I$ (A.8).

$$
R=\frac{R_{I} R_{I I}}{R_{I}+R_{I I}}
$$

Specific resistance or resistivity is equal to $r=R l_{1} l_{2} / l_{3}$, where $l_{3}$ is the length of the unit along the direction of electrical current propagation and $l_{1} l_{2}$ is the area of the unit perpendicular to the current propagation. The formulas for resistance of the unit with isolated pore geometry are presented in Table A.1, the derivation was carried out similarly.

The partially interconnected pore model, its subdivision into rectangular elements of matrix, pore and contact region and the equivalent network is presented in Fig. A.1(c). The contact region of the zero-generation is filled with the 1stgeneration of units $\mathrm{A}$. The contact region element in the equivalent network is replaced with the equivalent network for the unit A. Calculation of the resistivity is an iterative process for the partially interconnected pore model. First, resistivity of the unit $\mathrm{B}$ is calculated. Then, resistivity of the zero-generation is calculated using the formula for unit A where the resistivity of the contact region equals to the calculated resistivity of unit B. 
Elastic moduli $M=\lambda+2 \mu$ and $\mu$ were calculated by Spangenberg (1998) for the unit B, $\lambda$ and $\mu$ are Lamé constants. The elastic modules for unit A can be derived similarly. The formulas for elastic moduli are presented in Table A.1. For calculations, elastic moduli $M_{c}$ and $\mu_{c}$ of the contact region of the smallest generation are assumed to be equal to elastic moduli of the matrix material $M_{m}$ and $\mu_{m}$. Compressional and shear wave velocity can be calculated as the formulas and the density (A.2) for the model as $V_{p}=\sqrt{M / \rho}$ and $V_{s}=\sqrt{\mu / \rho}$.

\section{References}

Ahrens, T. J. (ed.), Mineral Physics and Crystallography. A Handbook of Physical Constants, American Geophysical Union, 354 pp., 2000.

Clark, S. P. (ed.), Handbook of Physical Constants, The Geological Society of America, 589 pp., 1966.

Hyndman, R. D. and P. M. Shearer, Water in the lower continental crust: Modeling magnetotelluric and seismic reflection results, Geophys. J. Int., 98, 343-365, 1989.

Iio, Y., A possible generating process of the 1995 Southern Hyogo Prefecture earthquake, J. Seism. Soc. Japan, 49, 103-112, 1996 (in Japanese with English abstract).

Iio, Y. and Y. Kobayashi, A physiscal understanding of large intraplate earthquakes, Earth Planets Space, 54(11), 1001-1004, 2002.

Imanishi, K., H. Ito, Y. Kuwahara, Y. Mamada, T. Yokokura, N. Kano, K. Yamaguchi, and A. Tanaka, Deep structure of the Nagamachi-Rifu fault from small aperture seismic array observations, Earth Planets Space, 54(11), 1033-1038, 2002.

Jones, A. G., Waves of the future: Superior inferences from collocated seismic and electromagnetic experiments, Tectonophysics, 286, 273298, 1998.

Kennett, B. L. N., S. Widiyantoro, and R. D. van der Hilst, Joint seismic tomography for bulk sound and shear wave speed in the Earth's mantle, J. Geophys. Res., 103(B6), 12,469-12,493, 1998.

Masters, G., G. Laske, H. Bolton, and A. Dziewonski, The relative behavior of shear velocity, bulk sound speed, and compressional velocity in the mantle: Implications for chemical and thermal structure, in Earth's Deep Interior: Mineral Physics and Tomography from the Atomic to the Global Scale, Geophys. Monogr. Ser., vol. 117, edited by S. Karato et al., pp. 63-87, AGU, Washington D. C., 2000.

Nakajima, J., Temperature and fluid distributions in the northeastern Japan arc inferred from seismic velocity and anisotropy structures, Doctoral dissertation, Tohoku University, Sendai, Japan, 146 pp., 2002 (in Japanese).

Nakajima, J., A. Hasegawa, S. Horiuchi, K. Yoshimoto, T. Yoshida, and N. Umino, Crustal heterogeneity around the Nagamachi-Rifu fault, northeastern Japan, as inferred from travel-time tomography, Earth Planets
Space, 2004 (submitted).

Nakamura, A., Y. Asano, and A. Hasegawa, Estimation of deep fault geometry of the Nagamachi-Rifu fault from seismic array observations, Earth Planets Space, 54(11), 1027-1032, 2002.

Nishimura, T., T. Sagiya, and S. Miura, Crustal deformation around the Nagamachi-Rifu fault zone and its vicinity (central Tohoku), northeastern Japan, observed by a continuous GPS network, Zisin (J. Seismol. Soc. Jpn.), 56(4), 2004 (in Japanese).

Ogawa, Y., M. Mishina, Y. Honkura, K. Takahashi, and S. B. Tank, Electromagnetic Imaging of the Nagamachi-Rifu Fault and Its Deep Extension, The Second International Symposium on Slip and Flow Processes in and below the Seismogenic Region, 2004.

Pervukhina, M., Y. Kuwahara, and H. Ito, Modelling of elastic and electrical properties of dry and saturated rock. XXIII General Assembly of International Union of Geodesy and Geophysics, June 30-July 11, Sapporo, Japan, 2003.

Pervukhina, M., Y. Kuwahara, and H. Ito, Fractal network and mixture models for elastic and electrical properties of porous rock, in Fractal Behavior of the Earth System, Springer Verlag Publisher, Germany, 2004 (accepted).

Sato, H., T. Imaizumi, T. Yoshida, H. Ito, and A. Hasegawa, Tectonic evolution and deep to shallow geometry of Nagamachi-Rifu Active Fault System, NE Japan, Earth Planets Space, 54(11), 1039-1043, 2002.

Simpson, F. and M. Warner, Coincident magnetotelluric, P-wave and Swave images of the deep continental crust beneath the Weardale granite, NE England: Seismic layering, low conductance and implications against the fluids paradigm, Geophys. J. Int., 133, 419-434, 1998.

Spangenberg, E., A fractal model for physical properties of porous rock: Theoretical formulations and applications, J. Geophys. Res., 103(B6), 12,269-12,289, 1998.

Takei, Y., Constitutive mechanical relations of solid-liquid composites in terms of grain-boundary contiguity, J. Geophys. Res., 103, 18,183$18,203,1998$.

Takei, Y., Effect of pore geometry on $V_{p} / V_{s}$ : From equilibrium geometry to crack, J. Geophys. Res., 107(B2), 10.1029/2001JB000522, 2002.

Tanaka, A. and Y. Ishikawa, Temperature distribution and focal depth of NE Japan, Earth Planets Space, 54(11), 1109-1113, 2002.

Umino, N., H. Ujikawa, S. Hori, and A. Hasegawa, Distinct S-wave reflectors (bright sports) detected beneath the Nagamachi-Rifu fault, NE Japan, Earth Planets Space, 54(11), 1021-1026, 2002.

Watson, E. B. and J. M. Brenan, Fluids in lithosphere, 1. Experimentally determined wetting characteristics of $\mathrm{CO}_{2}-\mathrm{H}_{2} \mathrm{O}$ fluids and their implications for fluid transport, host-rock physical properties, and fluid inclusion formation, Earth and Planetary Science Letters, 85, 497-515, 1987.

M. Pervukhina (e-mail: marina-pervuhkina@aist.go.jp), Y. Kuwahara, and $\mathrm{H}$. Ito 